\title{
Antimicrobial resistance point-of-care testing for gonorrhoea treatment regimens: cost-effectiveness and impact on ceftriaxone use of five hypothetical strategies compared with standard care in England sexual health clinics
}

Emma M Harding-Esch ${ }^{1,2}$, Susie E Huntington ${ }^{3}$, Michael J Harvey ${ }^{3}$, Georgie Weston ${ }^{3}$, Claire E Broad ${ }^{1}$, Elisabeth J Adams ${ }^{3}$, S Tariq Sadiq ${ }^{1,2,4}$

1. Applied Diagnostic Research and Evaluation Unit, Institute for Infection and Immunity, St George's University of London, London, United Kingdom

2. National Infection Service, Public Health England, London, United Kingdom

3. Aquarius Population Health, London, United Kingdom

4. St George's University Hospitals NHS Foundation Trust, London, United Kingdom

Correspondence: S Tariq Sadiq (ssadiq@sgul.ac.uk)

Background: Widespread ceftriaxone antimicrobial resistance (AMR) threatens Neisseria gonorrhoeae (NG) treatment, with few alternatives available. AMR pointof-care tests (AMR POCT) may enable alternative treatments, including abandoned regimens, sparing ceftriaxone use. We assessed cost-effectiveness of five hypothetical AMR POCT strategies: A-C included a second antibiotic alongside ceftriaxone; and $D$ and $E$ consisted of a single antibiotic alternative, compared with standard care (SC: ceftriaxone and azithromycin). Aim: Assess costs and effectiveness of AMR POCT strategies that optimise NG treatment and reduce ceftriaxone use. Methods: The five AMR POCT treatment strategies were compared using a decision tree model simulating 38,870 NG-diagnosed England sexual health clinic (SHC) attendees; A micro-costing approach, representing cost to the SHC (for 2015/16), was employed. Primary outcomes were: total costs; percentage of patients given optimal treatment (regimens curing NG, without AMR); percentage of patients given non-ceftriaxone optimal treatment; cost-effectiveness (cost per optimal treatment gained). Results: All strategies cost more than SC. Strategy B (azithromycin and ciprofloxacin (azithromycin preferred); dual therapy) avoided most suboptimal treatments $(n=48)$ but cost most to implement (GBP 4,093,844 (EUR $5,474,656)$ ). Strategy D (azithromycin AMR POCT; monotherapy) was most cost-effective for both cost per optimal treatments gained (GBP 414.67 (EUR 554.53)) and per ceftriaxone-sparing treatment (GBP 11.29
(EUR 15.09)) but with treatment failures $(n=34)$ and suboptimal treatments $(n=706)$. Conclusions: AMR POCT may enable improved antibiotic stewardship, but require net health system investment. A small reduction in test cost would enable monotherapy AMR POCT strategies to be cost-saving.

\section{Introduction}

Antimicrobial resistance (AMR) has developed to every class of antibiotic used for treatment of the bacterial sexually transmitted infection (STI) Neisseria gonorrhoeae (NG) [1], with increasing reports of multidrugresistant strains [2]. NG, the second most prevalent bacterial STI globally [3], is associated with serious long-term reproductive health complications if left untreated.

World Health Organization (WHO) guidelines [4] recommend a treatment regimen that treats at least $95 \%$ of circulating NG strains, as monitored through antibiotic surveillance programmes such as Public Health England's national Gonococcal Resistance to Antimicrobials Surveillance Programme (GRASP) [1]. Dual therapy with ceftriaxone and azithromycin is recommended in Europe [5], and was in the United Kingdom (UK) until 2019 [6] when it was replaced with $1 \mathrm{~g}$ ceftriaxone monotherapy due to the emergence of azithromycin resistance [7]. AMR to ceftriaxone, an extended-spectrum cephalosporin, is the most urgent 
Summary of antimicrobial resistance point-of-care test strategies

\section{Standard care}

Standard care with dual therapy of intramuscular ceftriaxone (500 mg) and oral azithromycin ( $1 \mathrm{~g}$ single dose).

Dual therapy, including ceftriaxone

A) AMR POCT for ciprofloxacin resistance only. Infections identified as not resistant to ciprofloxacin are given oral ciprofloxacin $(500 \mathrm{mg})$ plus ceftriaxone $(500 \mathrm{mg})$. Infections identified as ciprofloxacin resistant are given SC.

B) Dual AMR POCT for azithromycin and ciprofloxacin resistance. If no azithromycin resistance is identified, SC is given. If azithromycin resistant, ciprofloxacin $(500 \mathrm{mg})$ and ceftriaxone $(500 \mathrm{mg})$ are given unless there is ciprofloxacin resistance, in which case ceftriaxone $(500 \mathrm{mg})$ is given alone.

C) Dual AMR POCT for ciprofloxacin and azithromycin resistance. If no ciprofloxacin resistance is identified, ciprofloxacin (50o $\mathrm{mg}$ ) and ceftriaxone $(500 \mathrm{mg}$ ) are given. If ciprofloxacin resistant, $\mathrm{SC}$ is given, unless there is also azithromycin resistance, when ceftriaxone $(500 \mathrm{mg})$ is given alone.

\section{Monotherapy optimisation}

D) AMR POCT for azithromycin resistance. If no azithromycin resistance is identified, azithromycin ( $2 \mathrm{~g}$ ) is given. If azithromycin resistant, ceftriaxone $(500 \mathrm{mg})$ and ciprofloxacin $(500 \mathrm{mg})$ dual therapy is given. If the AMR POCT incorrectly shows no resistance (false negative for AMR), it is assumed the treatment fails. The treatment failure would be identified in the test-of-cure and the patient would then receive $500 \mathrm{mg}$ ceftriaxone.

E) AMR POCT for ciprofloxacin. If no ciprofloxacin resistance is identified, $500 \mathrm{mg}$ ciprofloxacin monotherapy is given. If ciprofloxacin resistant, SC is given. If the AMR POCT incorrectly shows no resistance, monotherapy is assumed to fail, the patient returns and receives $500 \mathrm{mg}$ ceftriaxone alone.

AMR: amtimicrobial resistance; POCT: point-of-care test; SC: standard care.

threat $[8,9]$ with few practical alternatives immediately available if widespread resistance develops.

Rapid diagnostics have been identified as a key approach to tackling AMR [10]. Rapid tests are those that have a two-hour turnaround, whereas point-of-care tests (POCTs) enable the test to be conducted, results obtained and treatment performed in the same clinical visit [11]. A principal feature of an NG AMR diagnostic is to assess antibiotic susceptibility at the time of NG diagnosis. A test that combines both NG diagnosis and AMR prediction at the point-of-care (AMR POCT) would allow the selection of appropriate treatment regimens for considerable numbers of NG infections, including safe use of antimicrobials which have been abandoned for widespread use due to circulating resistance, but which would be effective for a substantial proportion of infections [12]. For example, in the UK in 2018, $60 \%$ of NG infections were susceptible to ciprofloxacin, $90 \%$ to azithromycin and $88 \%$ to penicillin [1]. The ability to use these antibiotics to treat NG may in turn reduce AMR selection pressure on ceftriaxone [13].

Rapid tests are already being used for NG in some sexual health clinics (SHC) [14]. While laboratory-based NG fluoroquinolone susceptibility tests exist [15], rapid NG AMR tests are in development and being clinically evaluated. One such test is an NG fluoroquinolone susceptibility AMR POCT, developed within the Precise Study [16] using the io platform (Binx Health Limited (formerly Atlas Genetics), Boston, United States (US)) already CE-marked for Chlamydia trachomatis (CT) detection $[12,17]$. Costs and short-term clinical impacts of these tests are used in procuring sexual health services provision for a region (known as sexual health commissioning in England) and adoption into SHC decisionmaking [18].

In this analysis, we assessed the cost-effectiveness in English SHC of five hypothetical AMR POCT strategies for the treatment of NG, which enable use of ciprofloxacin and/or azithromycin, either alongside, or as an alternative to, ceftriaxone. Potential diagnostic resistance-determinants of these antibiotics are small in number (gyrA for ciprofloxacin; $23 S$ rRNA and mtrCDE transporter for azithromycin), are relatively well-understood, and their absence predictive of susceptibility (particularly for ciprofloxacin). The development of molecular AMR POCTs for detection of these determinants are thus technically feasible and therefore more likely to be immediately available [19-21].

\section{Methods}

\section{Model structure}

We compared standard care (SC) for NG treatment in the UK (at the time of investigation, ceftriaxone $500 \mathrm{mg}$ and azithromycin $1 \mathrm{~g}$ dual therapy [6]) with five different AMR POCT strategies (Box, Supplementary Figure S1), where the AMR POCT was used as a reflex test to inform antibiotic selection irrespective of which test was used to diagnose NG initially. The AMR POCT strategies were chosen to either facilitate optimised choice 
of a second antibiotic alongside ceftriaxone (dual therapy), or enable a single antibiotic alternative to ceftriaxone (monotherapy) (Box and Table 1).

The rationale for dual therapy strategies is based on the assumption that combination therapy is more effective at preventing emergence or spread of AMR and thereby preserves the use of ceftriaxone. The rationale for the monotherapy strategies is that an AMR POCT enables effective treatment of the known resistance profile, sparing the use of ceftriaxone [22].

Each strategy consisted of a series of intended treatment regimens, contingent on the results of the AMR POCT used. For example, in strategy B, the earliest intended treatment regimen was SC, where the AMR POCT indicated azithromycin resistance; the second intended treatment regimen was ceftriaxone and ciprofloxacin, where the AMR POCT then indicated ciprofloxacin resistance; the third intended treatment regimen was ceftriaxone monotherapy.

A decision tree model was constructed using TreeAge Pro version 2017 (TreeAge Software, Williamstown, United States (US)) to simulate a hypothetical cohort of 38,870 NG-diagnosed SHC attendees (21,915 men who have sex with men (MSM), 8,488 women and 8,467 men who have sex with women (MSW)), representing the total number of NG diagnoses in England $\mathrm{SHC}$ in 2015, obtained from the genitourinary medicine clinical activity dataset national surveillance data (GUMCAD) [23]. Our assumptions regarding AMR POCT use meant the model could not be used when considering presumptive treatment, e.g. for sexual contacts of NG-positive patients initially negative by microscopy but subsequently positive by nucleic acid amplification testing (NAAT). Approximately $10 \%$ of individuals diagnosed with NG are in contacts [24] but the epidemiological breakdown of these patients (e.g. women, MSW, MSM) and the nature of their NG diagnoses (e.g. microscopy negative and NAAT positive) is not reported. Therefore, contacts could not be removed from the hypothetical cohort.

Key model assumptions include: $100 \%$ compliance with test protocols; all patients entering the model are NG true-positives; dual AMR POCT results are available simultaneously; and there is no ceftriaxone resistance data (supported by England's GRASP [1]) so patients with monotherapy treatment failure would return and be successfully treated with ceftriaxone only. Model assumptions are provided in Supplementary Table S1.

\section{Outcomes}

We aimed to assess the costs and effectiveness of these AMR POCT strategies to optimise treatment regimen choice and reduce selection pressure on ceftriaxone. The primary outcomes were the total costs (2015/16 GBP(EUR)), the percentage of people given optimal treatment, and the percentage of people given non-ceftriaxone optimal treatment. Optimal treatment was defined as one which cured NG and did not contain an antibiotic against which there was resistance. Model definitions are provided in Supplementary Table S2. These data were used to calculate incremental cost-effectiveness ratios (ICER, see equation) for the cost per additional optimal treatment gained and the cost per additional ceftriaxone treatment avoided. This was chosen as the measure of cost-effectiveness rather than other measures, such as cost per Quality Adjusted Life Years (QALY), because little data exist on the consequence of optimal vs suboptimal NG treatment on long-term outcomes, such as mortality or lifetime costs.

$$
I C E R=\frac{\text { Cost }_{B}-\text { Cost }_{A}}{\text { Effectiveness }_{B}-\text { Effectiveness }_{A}}
$$

A: standard care; B: antimicrobial resistance point-ofcare test strategy; ICER: incremental cost-effectiveness ratio.

ICERs were calculated for two types of effectiveness: optimal treatments and ceftriaxone treatments avoided.

Secondary outcomes were the percentage of people given a missed earlier intended treatment regimen (MEITR), and the percentage of people failing treatment due to resistance. MEITR was defined as the use of a treatment regimen which cured NG, but where an earlier intended treatment regimen would have provided optimal treatment because susceptible infections had been misclassified as resistant by the AMR POCT. MEITRs were independent of treatment effectiveness.

\section{Treatment}

AMR POCT strategy treatment regimens were developed with input from three senior clinicians at St George's University Hospitals NHS Foundation Trust, London, who outlined current and hypothetical AMR POCT patient pathways (Supplementary Figure S1). The purpose of the work was to determine AMR POCT strategy for short-term clinical impacts, because these are the data used for sexual health service provisioning and decisionmaking for adoption into SHC [18]. Furthermore, progression to longer-term clinical impacts from suboptimally treated infection is poorly defined [25]. Therefore, the time horizon was that of initial patient treatment. Complications associated with STIs, such as pelvic inflammatory disease (PID) in women, and adverse drug events associated with treatment were not considered.

\section{Model parameters}

Model epidemiology parameters are presented in Table 2 , and cost parameters in Table 3 and Supplementary Table S3. The hypothetical AMR POCT sensitivity and specificity were based on other NAAT-based rapid and POC tests [26-28], and altered in sensitivity analyses. Antibiotic resistance prevalences were obtained from national surveillance of SHC attendees (GRASP, 2017) 
TABLE 1

Summary of antimicrobial point-of-care test strategies

\begin{tabular}{|c|c|c|c|c|c|c|c|c|c|c|}
\hline \multirow{3}{*}{\begin{tabular}{|l} 
Strategy \\
Strategy A \\
\end{tabular}} & \multicolumn{3}{|c|}{$\begin{array}{l}\text { Antibiotic(s) for which } \\
\text { resistance is tested }\end{array}$} & \multicolumn{7}{|c|}{ Intended treatment regimen based on test result } \\
\hline & A & + & B & \multicolumn{3}{|c|}{ No resistance to $A$} & \multicolumn{3}{|c|}{ Resistance to A } & $\begin{array}{c}\text { Resistance to } \\
\mathrm{A}+\mathrm{B}\end{array}$ \\
\hline & Ciprofloxacin & NA & NA & Ciprofloxacin & + & Ceftriaxone & Azithromycin & + & Ceftriaxone & NA \\
\hline Strategy B & Azithromycin & + & Ciprofloxacin & Azithromycin & + & Ceftriaxone & Ciprofloxacin & + & Ceftriaxone & Ceftriaxone \\
\hline Strategy C & Ciprofloxacin & + & Azithromycin & Ciprofloxacin & + & Ceftriaxone & Azithromycin & + & Ceftriaxone & Ceftriaxone \\
\hline Strategy D & Azithromycin & NA & NA & Azithromycin $^{\mathrm{a}, \mathrm{b}}$ & NA & NA & Ciprofloxacin & + & Ceftriaxone & NA \\
\hline Strategy E & Ciprofloxacin & $\mathrm{NA}$ & NA & Ciprofloxacin & NA & NA & Azithromycin & + & Ceftriaxone & NA \\
\hline
\end{tabular}

NA: not applicable.

a Dose given: $2 \mathrm{~g}$.

${ }^{b}$ If incorrect test result and treatment fails, ceftriaxone is given.

Bold font indicates standard care antibiotics, i.e. azithromycin and ceftriaxone dual therapy.

Unless otherwise stated, doses are: ceftriaxone $500 \mathrm{mg}$; azithromycin $1 \mathrm{~g}$; ciprofloxacin $500 \mathrm{mg}$.

[29]. GRASP is England's national sentinel surveillance system that detects and monitors AMR in NG and records potential treatment failures. As the time horizon was that of initial patient treatment, discounting rates were not applied.

A micro-costing approach was employed, considering only costs incurred to the healthcare provider (i.e. $\mathrm{SHC}$ ). Costs to those procuring sexual health services provision, or to health systems as a whole, were not considered. Costs were estimated by adapting an existing model [30] and included: laboratory equipment; POCTs and antibiotics; AMR POCTs; and NG treatment implementation (e.g. staff time and consumables, including partner notification and health promotion) (Supplementary Table S3). It was assumed the AMR POCTs produced results in 30 min (maximum acceptable POCT run-time for service users [31,32]) and that in all strategies, NG-positive samples would still be sent to the laboratory for culture and phenotypic resistance testing. Costs are given in 2015/16 prices (GBP (EUR)) and inflated when based on old estimates [33]. Antibiotic prices were extracted from the British National Formulary (BNF) website (September 2016), with the cheapest formulation being used including non-proprietary costs where available [34]. Initial costs of diagnosing NG were not considered as people only entered the model after an NG diagnosis. The cost of implementing a change to clinical practice was also not considered.

\section{Sensitivity analyses}

We conducted one-way analyses using TreeAge Pro version 2017 (TreeAge Software) and R software version 3.5.0 (R Foundation, Vienna, Austria), for each of the model parameters by varying them independently at the ends of their ranges to examine the effect on the primary outcome (Table 2). These analyses identified which model parameters results were most sensitive to. Each sensitivity analysis compared one of the five
AMR POCT strategies with SC, across three population groups (women, MSW, and MSM). Probabilistic sensitivity analyses (PSA) were not performed because our analysis was a cost-effectiveness analysis with the outcome as cost per event avoided, rather than a cost acceptability or cost utility analysis exploring the likelihood that the technology is cost-effective at different willingness to pay (WTP) thresholds. There is no commonly agreed WTP for our outcome, and therefore presenting PSA results would likely not have yielded additional beneficial information.

This report was written following the Consolidated Health Economic Evaluation Reporting Standards (CHEERS) checklist [35].

\section{Ethical statement}

As the only data included for this study were nationally published surveillance data from PHE, and no patient data were used, ethical approval was not required.

\section{Results}

Overall AMR POCT strategy costs, treatments used, and treatment outcomes compared with SC in all groups are presented in Table 4. Breakdowns by population group are presented in Supplementary Tables $\mathrm{S}_{4}, \mathrm{~S}_{5}$ and $\mathrm{S}_{6}$.

\section{Costs}

The cost of SC NG management was GBP 2,856,168 (EUR 3,819,524) for the total cohort (Table 4). All AMR POCT strategies cost more than SC, with dual therapy AMR POCT strategies more expensive than monotherapy strategies. Strategy D was the least expensive AMR POCT strategy, costing GBP 3,271,684 (EUR 4,375,189), $14.5 \%$ more than SC. Strategy B was the most expensive, costing GBP 4,093,844 (EUR 5,474,656), 43\% more than SC. This was consistent across all population groups (Supplementary Tables $\mathrm{S}_{4}, \mathrm{~S}_{5}$ and $\mathrm{S}_{6}$ ). 
TABLE 2

Epidemiology parameters used in the model for antimicrobial point-of-care test strategies

\begin{tabular}{|c|c|c|c|c|c|c|c|c|c|c|c|c|c|c|}
\hline \multirow{3}{*}{\multicolumn{2}{|c|}{ Variable }} & \multicolumn{6}{|c|}{ Percentage (\%) } & \multicolumn{6}{|c|}{ Number } & \multirow{3}{*}{$\begin{array}{l}\text { Comments } \\
\text { and } \\
\text { references }\end{array}$} \\
\hline & & \multicolumn{2}{|c|}{ MSM } & \multicolumn{2}{|c|}{ W } & \multicolumn{2}{|c|}{ MSW } & \multicolumn{2}{|r|}{ MSM } & \multicolumn{2}{|c|}{ w } & \multicolumn{2}{|c|}{ MSW } & \\
\hline & & $\begin{array}{c}\text { Base } \\
\text { case } \\
\text { value }\end{array}$ & $\begin{array}{l}\text { Range } \\
\text { (low, } \\
\text { high) }\end{array}$ & $\begin{array}{l}\text { Base } \\
\text { case } \\
\text { value }\end{array}$ & $\begin{array}{l}\text { Range } \\
\text { (low, } \\
\text { high) }\end{array}$ & $\begin{array}{c}\text { Base case } \\
\text { value }\end{array}$ & $\begin{array}{l}\text { Range } \\
\text { (low, } \\
\text { high) }\end{array}$ & $\begin{array}{l}\text { Base } \\
\text { case } \\
\text { value }\end{array}$ & $\begin{array}{l}\text { Range (low, } \\
\text { high) }\end{array}$ & $\begin{array}{c}\text { Base case } \\
\text { value }\end{array}$ & $\begin{array}{l}\text { Range } \\
\text { (low, } \\
\text { high) }\end{array}$ & $\begin{array}{c}\text { Base case } \\
\text { value }\end{array}$ & $\begin{array}{l}\text { Range (low, } \\
\text { high) }\end{array}$ & \\
\hline 1 & $\begin{array}{l}\text { Initial clinic } \\
\text { attendances }\end{array}$ & 56.4 & NA & 21.8 & NA & 21.8 & NA & 21,915 & NA & 8,488 & NA & 8,467 & NA & $\begin{array}{l}\text { GUMCAD, } \\
2015 \text { [23] }\end{array}$ \\
\hline 2 & $\begin{array}{l}\text { Resistance to } \\
\text { azithromycin }^{\text {a }}\end{array}$ & $4 \cdot 7$ & $3.3-6.1$ & 2.7 & $1.9-3.5$ & $5 \cdot 3$ & $3.7-6.9$ & 1,030 & $723-1,337$ & 229 & $161-297$ & 449 & $313-584$ & $\begin{array}{c}\text { GRASP, } 2017 \\
{[29]}\end{array}$ \\
\hline 3 & $\begin{array}{l}\text { Resistance to } \\
\text { ceftriaxone }\end{array}$ & o & $0-0$ & o & $0-0$ & o & $0-0$ & 0 & $0-0$ & 0 & $0-0$ & o & $0-0$ & $\begin{array}{c}\text { GRASP, } 2017 \\
\text { [29] }\end{array}$ \\
\hline 4 & $\begin{array}{l}\text { Resistance to } \\
\text { ciprofloxacin }^{\mathrm{b}}\end{array}$ & 36.2 & $25.3-47.1$ & 20.1 & $14.1-26.1$ & 32.5 & $22.8-42.3$ & 7,933 & $5,544-10,322$ & 1,706 & $\begin{array}{c}1,197^{-} \\
2,215 \\
\end{array}$ & 2,752 & $1,930-3,582$ & $\begin{array}{c}\text { GRASP, } 2017 \\
{[29]}\end{array}$ \\
\hline 5 & $\begin{array}{l}\text { Sensitivity of } \\
\text { AMR POCT }\end{array}$ & 98 & $90-100$ & 98 & $90-100$ & 98 & $90-100$ & NA & NA & NA & NA & NA & NA & Assumption \\
\hline 6 & $\begin{array}{l}\text { Specificity of } \\
\text { AMR POCT }\end{array}$ & 99 & $90-100$ & 99 & $90-100$ & 99 & $90-100$ & NA & NA & NA & NA & NA & NA & Assumption \\
\hline
\end{tabular}

AMR: antimicrobial resistance; GUMCAD: genitourinary medicine clinical activity dataset; GRASP: gonococcal resistance to antimicrobial surveillance programme; MSM: men who have sex with men; MSW: men who have sex with women; NA: not applicable; POCT: point-of-care test; W: women.

a The azithromycin resistance ranges were extended further to $1-10 \%$ for all population groups in one-way azithromycin resistance analysis so that the effect of more extreme values could be explored.

${ }^{b}$ The ciprofloxacin resistance ranges were extended further to $0-50 \%$ in one-way ciprofloxacin resistance analysis so that the effect of more extreme values could be explored.

\section{Optimal treatment}

All AMR POCT strategies provided more optimal treatments than SC, in all population groups. Strategy B provided most optimal $(n=38,822)$ and least suboptimal $(n=48)$ treatments. Strategies $A$ and E equally provided the least optimal treatments and the most suboptimal $(n=813)$ (see Table 4 and Supplementary Tables $\mathrm{S}_{4}, \mathrm{~S}_{5}$ and $\mathrm{S} 6$ ).

\section{Ceftriaxone-sparing treatments given}

Since all dual therapy strategies used ceftriaxone, only monotherapy strategies provided ceftriaxone-sparing options. Strategy D reduced ceftriaxone use by $95 \%$ compared with SC (Table 4).

\section{MEITRs given}

A MEITR refers to a treatment regimen being used when an earlier intended treatment regimen would have provided optimal treatment. In all population groups, the fewest were in Strategies A and E $(n=265)$, and $B$ $(n=267)$, and the most were in Strategy $C(n=912)$ (Table 4, Supplementary Tables S4, S5 and S6).

\section{Treatment failures}

There were some treatment failures in each monotherapy strategy due to false-susceptible AMR POCT results: strategy $D$ had $34 / 38,870$ ( $0.09 \%$ of treatments) and Strategy E had 248/38,870 (0.64\% of treatments) (Table 4). There were no treatment failures with SC or dual therapy strategies (A, B and C) because they all included ceftriaxone. This was consistent across all population groups (Supplementary Tables $\mathrm{S}_{4}, \mathrm{~S}_{5}$ and S6).

\section{Cost-effectiveness analysis}

The cost-effectiveness analysis (CEA) results are presented in Table 5. When avoidance of suboptimal treatments was considered, Strategy D was most cost-effective relative to SC, costing GBP 414.67 (EUR
554.53) per optimal treatment gained. Strategy A was least cost-effective overall, whereas Strategy B was the most-cost effective dual therapy strategy.

When avoidance of ceftriaxone use was considered, Strategy D was most cost-effective relative to SC, costing GBP 11.29 (EUR 15.09) per ceftriaxone-sparing treatment gained. These findings were consistent across all population groups.

\section{Sensitivity analyses}

In one-way sensitivity analyses, the following four parameters had the greatest impact on cost-effectiveness per optimal treatment gained for all AMR POCT strategies and across all population groups: prevalence of azithromycin resistance; AMR POCT sensitivity; prevalence of ciprofloxacin resistance; and the cost of single detection AMR POCT. In monotherapy strategies, the cost-effectiveness model was additionally sensitive to cost of clinical management (both with and without injection), cost of ceftriaxone, and AMR POCT specificity (for strategy D). The cost multiplier for a dual detection AMR POCT impacted on AMR POCT costeffectiveness for Strategies B and C. Tornado plots from these analyses are presented in Supplementary Figure S2.

For all strategies, variation of ICER in relation to azithromycin resistance prevalence was minimal until prevalence fell to or below 3\%, at which point it increased (Supplementary Figure S3). These rises in ICER were least for strategies $B$ and $D$. With the exception of strategy B where ICER were consistent for all population groups, these increases in ICER were most limited in women.

Variation in AMR POCT accuracy also showed similar patterns across all population groups. Apart from Strategy D, variation in specificity had very little effect on cost per optimal treatment gained. In contrast, as 
TABLE 3

Cost parameters used in the model for antimicrobial point-of-care test strategies

\begin{tabular}{|c|c|c|c|}
\hline \multirow{2}{*}{ Cost input } & \multicolumn{2}{|c|}{$\operatorname{Cost}^{\mathrm{a}}$} & \multirow{2}{*}{ Comments and references } \\
\hline & Base case value & Range (low-high) & \\
\hline $\begin{array}{l}\text { Management of NG (oral } \\
\text { medication/IM injection) }\end{array}$ & $\begin{array}{c}\text { GBP } 53.00 / \text { GBP } \\
62.74 \\
\text { (EUR } 70.88 \\
\text { / EUR 83.90) }\end{array}$ & $\begin{array}{c}\text { GBP } 37.10-68.90 / \text { GBP } \\
43.92-81.56 \\
\text { (EUR } 49.6-92.1 / \text { EUR } \\
58.73-109.07 \text { ) }\end{array}$ & ${ }^{\mathrm{b}}$ Adapted from previous model. Adams, 2014 [30] \\
\hline $\begin{array}{l}\text { Return visit due to treatment } \\
\text { failure }\end{array}$ & $\begin{array}{l}\text { GBP } 48.01 \\
(\text { EUR 64.20) }\end{array}$ & $\begin{array}{l}\text { GBP } 33.61-62.41 \\
\text { (EUR } 44.95-83.46)\end{array}$ & b,c Adapted from previous model. Adams, 2014 [30] \\
\hline Single AMR POCT & $\begin{array}{l}\text { GBP 29.00 } \\
\text { (EUR 38.78) }\end{array}$ & $\begin{array}{l}\text { GBP } 20.00-40.00 \\
\text { (EUR 26.75-53.49) }\end{array}$ & Estimate [47] \\
\hline Dual AMR POCT & $\begin{array}{l}\text { GBP } 31.90 \\
(\text { EUR } 42.66)\end{array}$ & $\begin{array}{l}\text { GBP } 29.00-58.00 \\
\text { (EUR } 38.78-77.56)\end{array}$ & $\begin{array}{c}\text { Estimate }-10 \% \text { more than price of single AMR POCT } \\
\text { (multiplier } 1.1 \text {, range } 1.0-2.0)\end{array}$ \\
\hline Dual AMR POCT & $\begin{array}{l}\text { GBP } 31.90 \\
(\text { EUR } 42.66)\end{array}$ & $\begin{array}{l}\text { GBP 22.00-44.00 } \\
\text { (EUR 29.42-58.84) }\end{array}$ & $\begin{array}{c}\text { Estimate }- \text { single AMR POCT is varied, multiplier } \\
\text { remains at } 1.1(10 \% \text { more than price of single AMR } \\
\text { POCT) }\end{array}$ \\
\hline Azithromycin $1 \mathrm{~g}^{\mathrm{d}}$ & $\begin{array}{l}\text { GBP } 1.16 \\
\text { (EUR 1.55) }\end{array}$ & $\begin{array}{l}\text { GBP } 0.81-1.51 \\
(\text { EUR 1.08-2.02) }\end{array}$ & BNF, $2016[34]$ \\
\hline Azithromycin $2 \mathrm{~g}^{\mathrm{d}}$ & $\begin{array}{l}\text { GBP } 2.32 \\
\text { (EUR 3.10) }\end{array}$ & $\begin{array}{l}\text { GBP 1.62-3.02 } \\
(\text { EUR 2.17-4.04) }\end{array}$ & BNF, $2016[34]$ \\
\hline Ceftriaxone $500 \mathrm{mg}$ e & $\begin{array}{l}\text { GBP 9.58 } \\
\text { (EUR 12.81) }\end{array}$ & $\begin{array}{l}\text { GBP } 6.71-12.45 \\
\text { (EUR 8.97-16.65) }\end{array}$ & BNF, $2016[34]$ \\
\hline Ciprofloxacin $500 \mathrm{mg}^{\mathrm{d}}$ & $\begin{array}{l}\text { GBP } 0.07 \\
\text { (EUR } 0.09 \text { ) }\end{array}$ & $\begin{array}{l}\text { GBP } 0.05-0.09 \\
(\text { EUR } 0.07-0.12)\end{array}$ & BNF, 2016 [34] \\
\hline
\end{tabular}

AMR: antimicrobial resistance; BNF: British National Formulary; IM: intramuscular; NG: Neisseria gonorrhoeae; POCT: point-of-care test. ${ }^{a}$ GBP costs were converted to EUR using a historic currency conversion of an average of 366 days from 1 July 2015 to 30 June 2016 [48]. For this time period, GBP $1=$ EUR 1.34, and EUR $1=$ GBP 0.75 .

b Includes staff time and consumables but not antibiotic costs. Costs were inflated to 2015/16 costs using the Hospital and Community Health Services (HCHS) Inflation Indices 2015 produced by the Personal Social Services Research Unit [33]. No data were available for inflation from $2014 / 15$ to $2015 / 16$ so it was assumed to be the same as between $2013 / 2014$ and $2014 / 15$. The United Kingdom hospital consumer price index for health services shows similar annual growth in this sector from 2014 (93.2 in 2013, 97.1 in 2014 and 100 in 2015), which validates this assumption [49]. GBP costs were converted to EUR using a historic currency conversion of an average of 366 days from 1 July 2015 to 30 June 2016 [48]. For this time period, GBP $1=$ EUR 1.34, and EUR $1=$ GBP 0.75 . A further breakdown of cost data are provided in Supplementary Table $\mathrm{S}_{3}$.

' Within the context of this model, treatment failure due to resistance to a monotherapy would result in a return visit. No repeat culture would be taken and no repeat diagnostic tests would occur. The patient would be successfully treated using ceftriaxone, administered via injection.

${ }^{d}$ Oral medication.

e Administered via intramuscular injection. The price quoted is for $1 \mathrm{~g}$ vial of ceftriaxone, the smallest non-proprietary vial available [34] - the remaining $500 \mathrm{mg}$ is then discarded.

sensitivity decreased to a minimum of $90 \%$, particularly towards the lower range, the cost per optimal treatment gained increased exponentially, except for strategy B where the relationship was linear. Strategy $B$ also had the smallest change in ICER between maximum (100\%) and minimum (90\%) sensitivity (maximum difference of GBP 169.21 (EUR 226.28) per optimal treatment gained in women) compared with other strategies where the difference was in the thousands. For Strategy D, change in sensitivity had little impact on cost per optimal treatment gained, whereas when specificity decreased to below around $95.5 \%$, the cost per optimal treatment gained started to increase exponentially. The sensitivity analyses are presented in Supplementary Figure S4.
The prevalence of ciprofloxacin resistance had very little effect on cost per optimal treatment gained in Strategies B, C and D (Supplementary Figure S5). For Strategies $A$ and $E$, as ciprofloxacin resistance increased from ca $20 \%$, there was an exponential increase in cost per optimal treatment gained for women only.

The relationship between ICER and cost of a single target AMR POCT was linear. Interestingly, as the cost of the single target AMR POCT increased, the two dualtarget AMR POCTs diverged, with strategy B costing less per optimal treatment gained relative to strategy c.

For the three single target AMR POCTs (A, D and E), reducing the cost of the test had the greatest impact 
Total costs, treatments used and treatment outcomes for standard care and antimicrobial resistance point-of-care test strategies: all groups $(\mathrm{n}=38,870)$

\begin{tabular}{|c|c|c|c|c|c|c|c|c|}
\hline \multirow[b]{2}{*}{ Strategy } & \multirow{2}{*}{ Total cost $\mathrm{t}^{\mathrm{a}}$} & \multicolumn{3}{|c|}{ Number of antibiotics used to treat NG } & \multirow{2}{*}{$\begin{array}{c}\text { Number } \\
\text { of optimal } \\
\text { treatments }\end{array}$} & \multirow{2}{*}{$\begin{array}{l}\text { Number of } \\
\text { suboptimal } \\
\text { treatments }\end{array}$} & \multirow{2}{*}{$\begin{array}{l}\text { Number } \\
\text { of MEITR }^{\mathrm{d}}\end{array}$} & \multirow{2}{*}{$\begin{array}{c}\text { Number of } \\
\text { treatment } \\
\text { failures }\end{array}$} \\
\hline & & Ceftriaxone & Azithromycin & Ciprofloxacin & & & & \\
\hline Standard care & $\begin{array}{l}\text { GBP 2,856,168 } \\
\text { (EUR 3,819,524) }\end{array}$ & 38,870 & 38,870 & 0 & 37,162 & 1,708 & NA & NA \\
\hline $\begin{array}{l}\text { A: Single POCT for } \\
\text { ciprofloxacin; dual } \\
\text { therapy }\end{array}$ & $\begin{array}{l}\text { GBP } 3,954,554 \\
\text { (EUR 5,288,385) }\end{array}$ & 38,870 & 12,408 & 26,462 & 38,057 & 813 & 265 & NA \\
\hline $\begin{array}{l}\text { B: Dual POCT for } \\
\text { azithromycin and } \\
\text { ciprofloxacin; dual } \\
\text { therapy }\end{array}$ & $\begin{array}{l}\text { GBP 4,093,844 } \\
\text { (EUR 5,474,656) }\end{array}$ & 38,870 & 36,825 & 1,373 & 38,822 & 48 & 267 & NA \\
\hline $\begin{array}{l}\text { C: Dual POCT for } \\
\text { ciprofloxacin and } \\
\text { azithromycin; dual } \\
\text { therapy }\end{array}$ & $\begin{array}{l}\text { GBP }_{4,066,498} \\
\text { (EUR } 5,438,086)\end{array}$ & 38,870 & 11,736 & 26,462 & 38,611 & 259 & 912 & NA \\
\hline $\begin{array}{l}\text { D: Single POCT } \\
\text { for azithromycin; } \\
\text { monotherapy }\end{array}$ & $\begin{array}{l}\text { GBP } 3,271,684 \\
\text { (EUR 4,375,189) }\end{array}$ & 2,080 & 36,825 & 2,045 & 38,164 & 706 & 372 & 34 \\
\hline $\begin{array}{l}\text { E: Single POCT } \\
\text { for ciprofloxacin; } \\
\text { monotherapy }\end{array}$ & $\begin{array}{l}\text { GBP }_{3,457,581} \\
\text { (EUR 4,623,788) }\end{array}$ & 12,656 & 12,408 & 26,462 & 38,057 & 813 & 265 & 248 \\
\hline
\end{tabular}

AMR: antimicrobial resistance; MEITR: missed earlier intended treatment regimen; NG: Neisseria gonorrhoeae; POCT: point-of-care test.

a GBP costs were converted to EUR using a historic currency conversion of an average of 366 days from 1 July 2015 to 30 June 2016 [48]. For this time period, GBP $1=$ EUR 1.34 , and EUR $1=$ GBP 0.75 .

b 'Optimal' refers to a treatment regimen which cures the NG infection and does not contain any antibiotic against which there is resistance.

c 'Suboptimal' refers to a treatment regimen which contains antibiotics against which there is NG resistance - if the treatment is a monotherapy it will result in treatment failure.

d 'Missed earlier intended treatment regimen' (MEITR) refers to a treatment regimen which cures the NG infection and does not contain any antibiotic against which there is resistance, but a treatment regimen was used when an earlier intended treatment regimen would have provided optimal treatment - a MEITR is due to a false-resistant AMR POCT result.

e 'Treatment failure' refers to failure to cure an NG infection due to resistance to an antibiotic given as monotherapy and is due to a falsesusceptible AMR POCT result.

on cost per treatment gained. Monotherapy strategies became cost-saving (ICER< o) for all population groups when AMR POCT cost was $\leq$ GBP 18 (24.07 EUR) for Strategy D, and S GBP 16 (EUR 21.40) for Strategy E (Supplementary Figure S6). Strategy B had lowest costs per additional optimal treatment for dual therapy strategies.

\section{Discussion}

We assessed the cost-effectiveness and impacts of deploying AMR POCTs for N. gonorrhoeae. All AMR POCT strategies assessed resulted in more optimal treatments compared with SC. Monotherapy AMR POCT strategies provided ceftriaxone-sparing options, with Strategy D reducing the use of ceftriaxone by $95 \%$. Both outcomes are important in promoting antibiotic stewardship by minimising risks of breakthrough with ceftriaxone-resistant circulating strains, and reducing selection pressure for resistance developing to ceftriaxone, respectively.

Our cost-effectiveness analysis adapted a previously published cost-effectiveness model of introducing a dual CT/NG POCT into a SHC $[28,30]$, and was populated using available published data, and where unavailable, using unpublished data and expert opinion.
By employing a decision tree model approach we could account for sufficient complexity without over-building. However, this approach is, in contrast to using a transmission dynamic model [36], unable to assess outcomes such as the impact AMR POCTs could have on re-infection in a previously treated patient, on population prevalence or burden of disease, or on AMR evolution.

Turner et al. have adapted the same CT/NG POCT costeffectiveness model we used for our analysis $[28,30]$ to analyse the potential clinical and overall economic impact of an NG AMR POCT [37]. While theirs was not a cost-effectiveness analysis, and different model assumptions and parameters from ours were used, they also demonstrated that AMR POCTs could lead to overall reductions in ceftriaxone use, but that introduction of AMR POCTs incurred increased costs. Using an individual-based dynamic transmission model that incorporated partner treatment and which was applied to a London MSM population, Zienkiewicz et al. [38] also demonstrated that AMR POCTs for NG ciprofloxacin sensitivity reduced ceftriaxone use, by $70 \%$ compared with the reference scenario. An individual-based model of molecular NG AMR test use compared with culture within an NG surveillance system in remote settings 
TABLE 5

Cost effectiveness analysis for standard care and antimicrobial resistance point-of-care test strategies

\begin{tabular}{|c|c|c|c|c|c|c|c|}
\hline Subgroup & Comparison & Total additional cost ${ }^{a}$ & $\begin{array}{l}\text { Additional cost } \\
\text { per patient }^{\mathrm{a}}\end{array}$ & $\begin{array}{l}\text { Number of optimal } \\
\text { treatments gained }\end{array}$ & $\begin{array}{c}\text { Additional cost per } \\
\text { optimal treatment } \\
\text { gained }^{\mathrm{a}}\end{array}$ & $\begin{array}{l}\text { Number of } \\
\text { ceftriaxone } \\
\text { treatments avoided }\end{array}$ & $\begin{array}{c}\text { Additional cost per } \\
\text { ceftriaxone-sparing } \\
\text { treatment }^{\mathrm{a}}\end{array}$ \\
\hline \multirow{5}{*}{ All } & AMR POCT A vs SC & $\begin{array}{l}\text { GBP } 1,098,386.00 \\
\text { (EUR 1,468,860.00) }\end{array}$ & $\begin{array}{l}\text { GBP } 28.26 \\
\text { (EUR 37.79) }\end{array}$ & 895 & $\begin{array}{l}\text { GBP 1,226.97 } \\
\text { (EUR 1,640.81) }\end{array}$ & o & Dominated \\
\hline & AMR POCT B vs SC & $\begin{array}{l}\text { GBP } 1,237,676.00 \\
\text { (EUR 1,655,131.00) }\end{array}$ & $\begin{array}{l}\text { GBP } 31.84 \\
\text { (EUR 42.58) }\end{array}$ & 1,660 & $\begin{array}{l}\text { GBP } 745.44 \\
\text { (EUR 996.87) }\end{array}$ & o & Dominated \\
\hline & AMR POCT C vs SC & $\begin{array}{l}\text { GBP } 1,210,330.00 \\
\text { (EUR 1,618,562.00) }\end{array}$ & $\begin{array}{l}\text { GBP } 31.14 \\
\text { (EUR 41.64) }\end{array}$ & 1,449 & $\begin{array}{l}\text { GBP } 835.39 \\
\text { (EUR 1,117.16) }\end{array}$ & 0 & Dominated \\
\hline & AMR POCT D vs SC & $\begin{array}{l}\text { GBP } 415,516.00 \\
\text { (EUR 555,665.30) }\end{array}$ & $\begin{array}{l}\text { GBP } 10.69 \\
\text { (EUR 14.30) }\end{array}$ & 1,002 & $\begin{array}{l}\text { GBP } 414.67 \\
\text { (EUR 554.53) }\end{array}$ & 36,790 & $\begin{array}{l}\text { GBP } 11.29 \\
\text { (EUR 15.09) }\end{array}$ \\
\hline & AMR POCT E vs SC & $\begin{array}{l}\text { GBP 601,414.00 } \\
\text { (EUR 804,264.80) }\end{array}$ & $\begin{array}{l}\text { GBP } 15.47 \\
\text { (EUR 20.69) }\end{array}$ & 895 & $\begin{array}{l}\text { GBP } 671.82 \\
\text { (EUR 898.42) }\end{array}$ & 26,214 & $\begin{array}{l}\text { GBP } 22.94 \\
\text { (EUR 30.68) }\end{array}$ \\
\hline \multirow{5}{*}{ MSM } & AMR POCT A vs SC & $\begin{array}{l}\text { GBP } 620,274.00 \\
\text { (EUR 829,486.10) }\end{array}$ & $\begin{array}{l}\text { GBP 28.30 } \\
\text { (EUR 37.85) }\end{array}$ & 499 & $\begin{array}{l}\text { GBP 1,242.13 } \\
\text { (EUR 1,661.09) }\end{array}$ & 0 & Dominated \\
\hline & AMR POCT B vs SC & $\begin{array}{l}\text { GBP 697,730.00 } \\
\text { (EUR 933,067.20) }\end{array}$ & $\begin{array}{l}\text { GBP }_{31.84} \\
\text { (EUR 42.58) }\end{array}$ & 1,001 & $\begin{array}{l}\text { GBP } 697.32 \\
\text { (EUR 932.52) }\end{array}$ & 0 & Dominated \\
\hline & AMR POCT C vs SC & $\begin{array}{l}\text { GBP } 683,317.00 \\
\text { (EUR 913,792.80) }\end{array}$ & $\begin{array}{l}\text { GBP } 31.18 \\
\text { (EUR 41.70) }\end{array}$ & 864 & $\begin{array}{c}\text { GBP } 790.97 \\
\text { (EUR 1,057.76) }\end{array}$ & 0 & Dominated \\
\hline & AMR POCT D vs SC & $\begin{array}{l}\text { GBP 235,532.00 } \\
\text { (EUR 314,974.50) }\end{array}$ & $\begin{array}{l}\text { GBP 10.75 } \\
\text { (EUR 14.38) }\end{array}$ & 568 & $\begin{array}{l}\text { GBP 414.38 } \\
\text { (EUR 554.15) }\end{array}$ & 20,676 & $\begin{array}{l}\text { GBP } 11.39 \\
\text { (EUR 15.23) }\end{array}$ \\
\hline & AMR POCT E vs SC & $\begin{array}{l}\text { GBP } 358,920.00 \\
\text { (EUR 479,980.00) }\end{array}$ & $\begin{array}{l}\text { GBP } 16.38 \\
\text { (EUR 21.90) }\end{array}$ & 499 & $\begin{array}{l}\text { GBP } 718.75 \\
\text { (EUR 961.18) }\end{array}$ & 13,842 & $\begin{array}{l}\text { GBP 25.93 } \\
\text { (EUR 34.68) }\end{array}$ \\
\hline \multirow{5}{*}{ MSW } & AMR POCT A vs SC & $\begin{array}{l}\text { GBP 239,316.00 } \\
\text { (EUR 320,034.80) }\end{array}$ & $\begin{array}{l}\text { GBP 28.26 } \\
\text { (EUR 37.79) }\end{array}$ & 248 & $\begin{array}{c}\text { GBP } 965.92 \\
\text { (EUR 1,291.72) }\end{array}$ & 0 & Dominated \\
\hline & AMR POCT B vs SC & $\begin{array}{l}\text { GBP 269,519.00 } \\
\text { (EUR 360,425.00) }\end{array}$ & $\begin{array}{l}\text { GBP } 31.83 \\
(\text { EUR } 42.57)\end{array}$ & 436 & $\begin{array}{l}\text { GBP } 617.60 \\
\text { (EUR 825.91) }\end{array}$ & 0 & Dominated \\
\hline & AMR POCT C vs SC & $\begin{array}{l}\text { GBP } 263,674.00 \\
\text { (EUR 352,608.50) }\end{array}$ & $\begin{array}{l}\text { GBP } 31.14 \\
\text { (EUR 41.64) }\end{array}$ & 391 & $\begin{array}{l}\text { GBP 674.71 } \\
\text { (EUR 902.28) }\end{array}$ & 0 & Dominated \\
\hline & AMR POCT D vs SC & $\begin{array}{l}\text { GBP 91,956.00 } \\
\text { (EUR 122,971.80) }\end{array}$ & $\begin{array}{l}\text { GBP } 10.86 \\
\text { (EUR 14.52) }\end{array}$ & 271 & $\begin{array}{l}\text { GBP } 339.59 \\
\text { (EUR 454.13) }\end{array}$ & 7,938 & $\begin{array}{l}\text { GBP 11.58 } \\
\text { (EUR 15.49) }\end{array}$ \\
\hline & AMR POCT E vs SC & $\begin{array}{l}\text { GBP } 132,108.00 \\
\text { (EUR 176,666.70) }\end{array}$ & $\begin{array}{l}\text { GBP } 15.60 \\
\text { (EUR 20.86) }\end{array}$ & 248 & $\begin{array}{l}\text { GBP } 533.21 \\
\text { (EUR 713.06) }\end{array}$ & 5,658 & $\begin{array}{l}\text { GBP 23.35 } \\
\text { (EUR 31.23) }\end{array}$ \\
\hline \multirow{5}{*}{ Women } & AMR POCT A vs SC & $\begin{array}{l}\text { GBP } 238,796.00 \\
\text { (EUR } 319,339.40)\end{array}$ & $\begin{array}{l}\text { GBP 28.13 } \\
\text { (EUR 37.62) }\end{array}$ & 148 & $\begin{array}{l}\text { GBP 1,612.62 } \\
\text { (EUR 2,156.54) }\end{array}$ & 0 & Dominated \\
\hline & AMR POCT B vS SC & $\begin{array}{l}\text { GBP } 270,428.00 \\
\text { (EUR } 361,640.60 \text { ) }\end{array}$ & $\begin{array}{l}\text { GBP } 31.86 \\
\text { (EUR 42.61) }\end{array}$ & 223 & $\begin{array}{l}\text { GBP } 1,210.74 \\
\text { (EUR 1,619.11) }\end{array}$ & 0 & Dominated \\
\hline & AMR POCT C vS SC & $\begin{array}{l}\text { GBP } 263,339.00 \\
\text { (EUR 352,160.50) }\end{array}$ & $\begin{array}{l}\text { GBP } 31.02 \\
\text { (EUR 41.48) }\end{array}$ & 194 & $\begin{array}{l}\text { GBP } 1,356.61 \\
\text { (EUR 1,814.18) }\end{array}$ & 0 & Dominated \\
\hline & AMR POCT D vs SC & $\begin{array}{l}\text { GBP } 88,028.00 \\
\text { (EUR 117,718.90) }\end{array}$ & $\begin{array}{l}\text { GBP 10.37 } \\
\text { (EUR 13.87) }\end{array}$ & 163 & $\begin{array}{l}\text { GBP } 540.55 \\
(\text { EUR 722.87) }\end{array}$ & 8,176 & $\begin{array}{l}\text { GBP 10.77 } \\
\text { (EUR 14.40) }\end{array}$ \\
\hline & AMR POCT E vs SC & $\begin{array}{l}\text { GBP }_{110,386.00} \\
\text { (EUR 147,618.10) }\end{array}$ & $\begin{array}{l}\text { GBP 13.00 } \\
\text { (EUR 17.38) }\end{array}$ & 148 & $\begin{array}{l}\text { GBP } 745.45 \\
\text { (EUR 996.88) }\end{array}$ & 6,714 & $\begin{array}{l}\text { GBP } 16.44 \\
\text { (EUR 21.99) }\end{array}$ \\
\hline
\end{tabular}

AMR: antimicrobial resistance; MSM: men who have sex with men; MSW: men who have sex with women; POCT: point-of-care test; SC: standard care.

${ }^{a}$ GBP costs were converted to EUR using a historic currency conversion of an average of 366 days from 1 July 2015 to 30 June 2016 [48]. For this time period, GBP $1=$ EUR 1.34, and EUR $1=$ GBP 0.75 .

A strategy is 'dominated' if it is more expensive and provides fewer/equivalent benefits. 
found that they substantially improved the timeliness of NG AMR detection, facilitating a faster change in recommended treatment, with potential for decreasing NG AMR impact on the wider population [39]. Fingerhuth et al. [36] developed a compartmental transmission model of antibiotic-sensitive and antibiotic-resistant NG to look at proportion of resistant infections and cases averted. They showed that the clinical pathway that included an AMR POCT resulted in the lowest proportion of resistant infections after 30 years, whereas the clinical pathway with a POCT that did not test for AMR resulted in the highest. They also noted that test diagnostic performance is key for AMR POCTs to have a beneficial public health impact. The potential public health impact of AMR POCTs was confirmed by Tuite et al., with AMR POCTs delaying the proportion of isolates reaching $>5 \%$ resistance compared with empirical treatment [40]. However, it was highlighted that the AMR POCT should test for resistance to multiple anitimicrobials, otherwise non-tested, resistant, strains will be selected for. Thus, continued surveillance, including culture, should be continued. Together, these health economic and modelling evaluations highlight the possible beneficial impacts of implementing AMR POCTs on reducing ceftriaxone use and decreasing NG AMR prevalence at the population level, but the design and implementation of the tests should also be carefully considered.

As with all mathematical models, several assumptions were made (Supplementary Table S1), including AMR POCT diagnostic accuracy - a necessity as these tests are currently in early phases of development $[16,41]$. Future performance estimates will need to consider two elements: predictive accuracies of any biomarkers used to detect AMR; the performance of platforms and chemistries used to detect them. Variations in both may independently affect outcomes.

Our analysis had some limitations. We used the most recent NG AMR data available from GRASP at the time [29], but AMR rates constantly change and, in the sensitivity analyses, AMR prevalence alterations had the greatest impact on AMR POCT cost-effectiveness (Supplementary Figure S2). This may limit the generalisability of our results as it is not possible to know future resistance profiles. However, the results should be generalisable to the ranges used in the sensitivity analyses. In addition, as AMR POCTs are still in development, some of the model's other epidemiological parameters will have changed by the time the AMR POCTs are available for use in routine practice, which may further limit the analyses' applicability in the longer term. This highlights the need to continually conduct analyses such as these, to enhance our ability to predict and understand future trends. Our anlayses are also limited to data from England, with results perhaps less generalisable to other countries. This will be exacerbated by the 2019 change to $1 \mathrm{~g}$ ceftriaxone monotherapy, further setting it aside from guidelines in other European countries [7]. Our model also did not consider NG-positive patients coinfected with another organisms, such as CT, which would affect patient pathways and treatment options. Additional factors not considered were costs associated with treating longterm NG infection sequelae [42], costs incurred outside of the SHC, and costs or cost-savings associated with changing clinical pathways in order to accommodate the AMR POCTs. Thus the time horizon for the costs and consequences was of initial patient treatment only.

Strategy B was most effective for avoiding suboptimal treatments but the most costly to implement. Strategy $D$ was the most cost-effective for both effectiveness outcomes (optimal treatments gained and ceftriaxone avoidance), but resulted in treatment failures, as well as nearly 15 -fold higher suboptimal treatments compared with Strategy B. Both strategies B and D enabled the re-use of ciprofloxacin, previously abandoned for the treatment of NG in the UK [6].

All AMR POCT strategies were more expensive than SC, with dual therapy AMR POCT strategies more expensive than monotherapy strategies, suggesting that shortterm net financial investments in AMR POCT adoption are required to gain long-term antimicrobial stewardship benefits. The O'Neill review of AMR [10] noted that accepting the initial expense of new test introduction may enable longer-term societal pay-offs by reducing infection rates and maintaining effective NG treatments. Interestingly, our sensitivity analysis suggested that even if AMR POCT costs were notably reduced, perhaps through production scale-up, dual therapy AMR POCT strategies would still not be cost-saving. However, a relatively small reduction to less than GBP 18 (EUR 24.07) per test would enable the monotherapy AMR POCT strategies to be cost-saving.

The monotherapy strategies resulted in treatment failures due to false susceptible AMR POCT results, although minimal relative to SC. Since we assumed ceftriaxone treated $100 \%$ of NG infections, there were no treatment failures for SC or dual therapy strategies. The most recent GRASP data suggest that ceftriaxone resistance remains low (no ceftriaxone resistance reported, although there is a reduction in susceptibility with $24.6 \%$ of isolates with minimum inhibitory concentrations (MICs) $\geq 0.03 \mathrm{mg} / \mathrm{L}$ in 2018 compared with $16.6 \%$ in 2017 [1]), but there are increasing concerns regarding international ceftriaxone-resistant strains [43-45]. This potentially undermines our assumption and the resulting lack of treatment failures from dual therapy AMR POCT strategies.

Most MEITRs (treatment regimen used when an earlier intended treatment regimen would have provided optimal treatment) were in Strategy $C$, and the least in Strategies A, B and E. Avoiding MEITRs is important because it maximises the ability to use ciprofloxacin (in Strategies A, C and E), or reduces the need for ceftriaxone use (Strategies $B$ and $D$ ). These numbers were small compared with actual patient numbers in whom 
a MEITR might be used if these AMR POCTs were available more generally. For example, using national surveillance data $[23,46]$, we estimated that over 25,000 of the 38,870 NG-diagnosed SHC patients assumed to have been treated with SC in 2015 would have had ciprofloxacin-susceptible NG. Strategies A and E would have enabled all, except 265 (Table 4 ), of these patients to be treated with ciprofloxacin, a 100 -fold reduction in these missed opportunities.

Since a MEITR is due to susceptible infections misclassified as resistant by the AMR POCT, test specificity is key. In sensitivity analyses of AMR POCT accuracy, Strategy D was the only strategy where cost per optimal treatment gained was affected by changes in specificity. In all other strategies, cost per optimal treatment gained increased as sensitivity decreased. This is because these strategies contained an AMR POCT that included ciprofloxacin testing, so resistance (20-36\%, dependent on population group [29]) was detected and optimal treatment could be given. In contrast, if AMR POCT sensitivity in these strategies fell, true ciprofloxacin-resistant cases were missed and the patient suboptimally treated. Strategy D, where the AMR POCT was for azithromycin only, was the only strategy where ciprofloxacin was given without resistance-testing as the specificity decreased, more patients received false-positive azithromycin resistance results and were treated with ciprofloxacin. Due to high ciprofloxacin resistance prevalence, this treatment was suboptimal in a large number of cases. Following the logic of the other strategies, if azithromyin resistance prevalence increased, cost per optimal treatment gained in Strategy D would become sensitive to both AMR POCT specifity and sensitivity.

Thus, prevalence of resistance has important implications for AMR POCT accuracy requirements and ICER of optimal treatments gained. In the azithromycin resistance sensitivity analyses, ICER increased when resistance fell below ca $3 \%$ (well below current UK azithromycin resistance prevalence, reported at ca $9.7 \%$ [1]), primarily because when azithromycin resistance is low, there is little value in testing for it (Strategies B, C and D) and there will be few treatment failures from background resistance (Strategies $A$ and E). In the ciprofloxacin resistance sensitivity analysis, an effect on ICER was only seen in women in strategies $A$ and $E$ (because of lower baseline ciprofloxacin resistance prevalence).

From a population-level antimicrobial stewardship public health perspective, increasing the number of suboptimal treatments may eventually lead to an increased number of resistant infections [36]. The relative public health importance of a smaller total number of suboptimal treatments with a few treatment failures vs a higher number of suboptimal treatments with no failures warrants further investigation, and could be included in future transmission model analyses. Furthermore, the long-term public health impact of preserving ceftriaxone use while increasing the risk of treatment failures from monotherapy strategies (vs maintaining ceftriaxone in the earlier intended treatment regimen with an increase in suboptimal treatments and no adequate treatment alternative), should also be investigated.

\section{Conclusion}

Once developed, AMR POCTs could have wide-ranging implications for clinical decisionmaking globally, including the potential re-use of antibiotics previously abandoned for the treatment of NG, ensuring the right treatment is given to the right person at the right time (precision medicine). Although it may be necessary to accept net health system investment to enable longerterm societal pay-offs by reducing infection rates and maintaining effective NG treatments, a relatively small reduction in test cost could enable some AMR POCT strategies to be cost-saving.

empirical study and modelling is required to optimise their use for public health benefit.

\section{Acknowledgements}

Funding statement: This work was supported by the National Institute for Health Research (NIHR) i4i Programme (https:// www.nihr.ac.uk/about-us/how-we-aremanaged/boards-andpanels/programme-boards436and-panels/invention-for-innovation/) [grant number II-LB-0214-20005]. The funder had no role in study design, data collection and analysis, decision to publish, or preparation of the manuscript.

\section{Conflict of interest}

$\mathrm{SEH}, \mathrm{MJH}, \mathrm{GW}$ and EJA are employees of Aquarius Population Health (APH) which reports grants on STI and POC research outside the submitted work from Innovate UK, Cepheid, St Georges University of London, Enigma Diagnostics, and AstraZeneca. EHE, CEB and STS are members of the Applied Diagnostic Research and Evaluation Unit at St George's, University of London, which has received funding from Binx Health, Alere, Cepheid, SpeeDx and Sekisui.

\section{Authors' contributions}

EMHE, SEH, MJH, CEB, EJA and STS designed the model. MJH and GW ran the model. EMHE, SHE, MJH and STS wrote the first draft of the paper. All authors edited the manuscript and read and approved the final version of the paper.

\section{References}

1. Public Health England (PHE). Antimicrobial resistance in Neisseria gonorrhoeae in England and Wales. Key findings from the Gonococcal Resistance to Antimicrobials Surveillance Programme (GRASP 2018). London: PHE; Oct 2019. Available from: https://assets.publishing.service.gov.uk/government/ uploads/system/uploads/attachment_data/file/834924/ GRASP_2018_report.pdf

2. Abraha M, Egli-Gany D, Low N. Epidemiological, behavioural, and clinical factors associated with antimicrobial-resistant gonorrhoea: a review. F100o Res. 2018;7:400. https://doi. org/10.12688/f10ooresearch.13600.1 PMID: 29636908

3. Rowley J, Vander Hoorn S, Korenromp E, Low N, Unemo M, Abu-Raddad LJ, et al. Chlamydia, gonorrhoea, trichomoniasis 
and syphilis: global prevalence and incidence estimates, 2016. Bull World Health Organ. 2019;97(8):548-562P. https://doi. org/10.2471/BLT.18.228486 PMID: 31384073

4. World Health Organization (WHO). Global action plan to control the spread and impact of antimicrobial resistance in Neisseria gonorrhoeae. Geneva: WHO; 2012. Available from: http://apps. who.int/iris/bitstream/10665/44863/1/9789241503501_eng. pdf

5. Bignell C, Unemo M, Radcliffe K, Jensen JS, Babayan K, Barton S, et al. , European STI Guidelines Editorial Board. 2012 European guideline on the diagnosis and treatment of gonorrhoea in adults. Int J STD AIDS. 2013;24(2):85-92. https://doi.org/10.1177/0956462412472837 PMID: 24400344

6. Bignell C, Fitzgerald M, Guideline Development Group. British Association for Sexual Health and HIV UK. UK national guideline for the management of gonorrhoea in adults, 2011. Int J STD AIDS. 2011;22(10):541-7. https://doi.org/10.1258/ ijsa.2011.011267 PMID: 21998172

7. Fifer H, Saunders J, Soni S, Sadiq ST, FitzGerald M. 2019. British Association for Sexual Health and HIV national guideline for the managementof infection with Neisseria gonorrhoeae (2019). British London: Association for Sexual Health and HIV. 2019. Available from: https://www. bashhguidelines.org/media/1208/gc-2019.pdf

8. Fifer H, Natarajan U, Jones L, Alexander S, Hughes G, Golparian $\mathrm{D}$, et al. Failure of Dual Antimicrobial Therapy in Treatment of Gonorrhea. N Engl J Med. 2016;374(25):2504-6. https://doi. org/10.1056/NEJMC1512757 PMID: 27332921

9. Low N, Unemo M. Molecular tests for the detection of antimicrobial resistant Neisseria gonorrhoeae: when, where, and how to use? Curr Opin Infect Dis. 2016;29(1):45-51. https://doi.org/10.1097/QC0.0000000000000230 PMID: 26658656

10. O'Neill J. Rapid Diagnostics: stopping unnecessary use of antibiotics. London: Review on Antimicrobial Resistance; Oct 2015. Available from: http://amr-review.org/sites/default/files/ Rapid\%20Diagnostics\%20-\%20Stopping\%20Unnecessary\%20 use $\% 200$ \% 20 Antibiotics.pdf

11. World Health Organization (WHO). Simple / Rapid tests. Geneva: WHO. [Accessed: Jul 2016]. Available from: http:// www.who.int/diagnostics_laboratory/faq/simple_rapid_tests/ en/

12. Sadiq ST, Mazzaferri F, Unemo M. Rapid accurate point-ofcare tests combining diagnostics and antimicrobial resistance prediction for Neisseria gonorrhoeae and Mycoplasma genitalium. Sex Transm Infect. 2017;93(S4):S65-8. https://doi. org/10.1136/sextrans-2016-053072 PMID: 28684610

13. Sadiq ST, Dave J, Butcher PD. Point-of-care antibiotic susceptibility testing for gonorrhoea: improving therapeutic options and sparing the use of cephalosporins. Sex Transm Infect. 2010;86(6):445-6. https://doi.org/10.1136/ sti.2010.044230 PMID: 20940156

14. Herbst de Cortina S, Bristow CC, Joseph Davey D, Klausner JD. A Systematic Review of Point of Care Testing for Chlamydia trachomatis, Neisseria gonorrhoeae, and Trichomonas vaginalis. Infect Dis Obstet Gynecol. 2016;2016:4386127. https://doi.org/10.1155/2016/4386127 PMID: 27313440

15. SpeeDx. ResistancePlus GC.Australia: SpeeDx. [Accessed: 1 Mar 2020]. Available from: https://plexpcr.com/ resistanceplus-gc/

16. National Institute for Health Research (NIHR). Invention for Innovation grant: A Point of Care Antimicrobial Resistance test for Neisseria gonorrhoeae and Mycoplasma genitalium infection. Ensuring accurate therapy and antibiotic stewardship in sexual health medicine. Reference: II-LB-0214-20005. Available from: https://nihr.opendatasoft.com/explore/ dataset/infonihr-open-dataset/table/?disjunctive. programme type\&disjunctive.project status\&disjunctive. programme\&disjunctive.rgn17nm\&disjunctive. pcon17nm\&disjunctive.ccg18nm\&disjunctive. ctry17cd\&disjunctive.funder\&disjunctive.acronym\&disjunctive. ctry17nm\&disjunctive.ccg19nm\&disjunctive. lad19nm\&disjunctive.pcon18nm\&q=antimicrobial+resistance

17. Harding-Esch EM, Cousins EC, Chow SC, Phillips LT, Hall $\mathrm{CL}$, Cooper N, et al. A 30-minute nucleic acid amplification point-of-care test for genital Chlamydia trachomatis infection in women: a prospective, multi-centre study of diagnostic accuracy. EBioMedicine. 2018;28:120-7. https://doi. org/10.1016/j.ebiom.2017.12.029 PMID: 29396306

18. Public Health England (PHE). Making it work. A guide to whole system commissioning for sexual health, reproductive health and HIV. London: PHE; Mar 2015. Available from: https://www. gov.uk/government/uploads/system/uploads/attachment data/file/408357/Making_it_work_revised_March_2015.p̄jf

19. Pond MJ, Hall CL, Miari VF, Cole M, Laing KG, Jagatia H, et al. Accurate detection of Neisseria gonorrhoeae ciprofloxacin susceptibility directly from genital and extragenital clinical samples: towards genotype-guided antimicrobial therapy. J Antimicrob Chemother. 2016;71(4):897-902. https://doi. org/10.1093/jac/dkv432 PMID: 26817487

20. Allan-Blitz LT, Humphries RM, Hemarajata P, Bhatti A, Pandor MW, Siedner MJ, et al. Implementation of a Rapid Genotypic Assay to Promote Targeted Ciprofloxacin Therapy of Neisseria gonorrhoeae in a Large Health System. Clin Infect Dis. 2017;64(9):1268-70. PMID: 28034887

21. Eyre DW, De Silva D, Cole K, Peters J, Cole MJ, Grad YH, et al. WGS to predict antibiotic MICs for Neisseria gonorrhoeae. J Antimicrob Chemother. 2017;72(7):1937-47. https://doi. org/10.1093/jac/dkxo67 PMID: 28333355

22. Unemo M, Bradshaw CS, Hocking JS, de Vries HJC, Francis SC, Mabey D, et al. Sexually transmitted infections: challenges ahead. Lancet Infect Dis. 2017;17(8):e235-79. https://doi. org/10.1016/S1473-3099(17)30310-9 PMID: 28701272

23. Public Health England (PHE). Sexually transmitted infections (STIS): annual data tables, 2006-2015. London: PHE. [Accessed: 1 Mar 2020]. Available from: https://www.gov.uk/government/statistics/ sexually-transmitted-infections-stis-annual-data-tables

24. Public Health England (PHE). Table 7: STI diagnoses \& partner notification, 2012 - 2015. London: PHE. [Accessed: 1 Mar 2020]. Available from: https://assets.publishing.service.gov. uk/government/uploads/system/uploads/attachment_data/ file/534562/2015_Table_7_STI_diagnoses___partner_ notification 2012-2015.pdf

25. Herzog SA, Heijne JC, Althaus CL, Low N. Describing the progression from Chlamydia trachomatis and Neisseria gonorrhoeae to pelvic inflammatory disease: systematic review of mathematical modeling studies. Sex Transm Dis. 2012;39(8):628-37. https://doi.org/10.1097/ OLQ.ob013e31825159ff PMID: 22801346

26. Gaydos CA, Van Der Pol B, Jett-Goheen M, Barnes M, Quinn $\mathrm{N}$, Clark C, et al. , CT/NG Study Group. Performance of the Cepheid CT/NG Xpert Rapid PCR Test for Detection of Chlamydia trachomatis and Neisseria gonorrhoeae. J Clin Microbiol. 2013;51(6):1666-72. https://doi.org/10.1128/ JCM.03461-12 PMID: 23467600

27. Harding-Esch EM, Fuller SS, Christine Chow SL, Nori AV, Harrison MA, Parker M, et al. Diagnostic accuracy of a prototype rapid chlamydia and gonorrhoea recombinase polymerase amplification assay: a multi-centre cross-sectional pre-clinical evaluation. Clin Microbiol Infect. 2019;25(3):380e1380.e7. http://dx.doi.org/ https://doi.org/10.1016/j. cmi.2018.06.003 PMID: 29906594

28. Turner KM, Round J, Horner P, Macleod J, Goldenberg S, Deol A, et al. An early evaluation of clinical and economic costs and benefits of implementing point of care NAAT tests for Chlamydia trachomatis and Neisseria gonorrhoea in genitourinary medicine clinics in England. Sex Transm Infect. 2014;90(2):104-11. https://doi.org/10.1136/ sextrans-2013-051147 PMID: 24273127

29. Public Health England (PHE). Surveillance of antimicrobial resistance in Neisseria gonorrhoeae in England and Wales. Key findings from the Gonococcal Resistance to Antimicrobials Surveillance Programme (GRASP). London: PHE; 2017. Available from: https://assets.publishing.service.gov.uk/government/ uploads/system/uploads/attachment_data/file/651636/ GRASP Report_2017.pdf

30. Adams EJ, Ehrlich A, Turner KM, Shah K, Macleod J, Goldenberg $S$, et al. Mapping patient pathways and estimating resource use for point of care versus standard testing and treatment of chlamydia and gonorrhoea in genitourinary medicine clinics in the UK. BMJ Open. 2014;4(7):eo05322. https://doi. org/10.1136/bmjopen-2014-005322 PMID: 25056977

31. Harding-Esch EM, Nori AV, Hegazi A, Pond MJ, Okolo O, Nardone $A$, et al. Impact of deploying multiple pointof-care tests with a 'sample first' approach on a sexual health clinical care pathway. A service evaluation. Sex Transm Infect. 2017;93(6):424-9. https://doi.org/10.1136/ sextrans-2016-052988 PMID: 28159916

32. Atkinson LM, Vijeratnam D, Mani R, Patel R. 'The waiting game': are current chlamydia and gonorrhoea near-patient/ point-of-care tests acceptable to service users and will they impact on treatment? Int J STD AIDS. 2016;27(8):650-5. https://doi.org/10.1177/0956462415591414 PMID: 26092579

33. Personal Social Services Research Unit (PSSRU). Unit Costs of Health and Social Care 2015. Kent: PSSRU; 2015. Available from: http://www.pssru.ac.uk/project-pages/unitcosts/2015/2015 [

34. National Institute for Health and Care Excellence (NICE) British National Formulary. (BNF). London: NICE. [Accessed 29 Sept 2016]. Available from: https://www.evidence.nhs.uk/ formulary/bnf/current 
35. Husereau D, Drummond M, Petrou S, Carswell C, Moher D, Greenberg D, et al. Consolidated Health Economic Evaluation Reporting Standards (CHEERS) statement. BMJ. 2013;346(mar25 1):f1049. PMID: 23529982

36. Fingerhuth SM, Low N, Bonhoeffer S, Althaus CL. Detection of antibiotic resistance is essential for gonorrhoea pointof-care testing: a mathematical modelling study. BMC Med. 2017;15(1):142. https://doi.org/10.1186/s12916-017-0881-X PMID: 28747205

37. Turner KM, Christensen H, Adams EJ, McAdams D, Fifer H, McDonnell A, et al. Analysis of the potential for point-of-care test to enable individualised treatment of infections caused by antimicrobial-resistant and susceptible strains of Neisseria gonorrhoeae: a modelling study. BMJ Open. 2017;7(6):e015447. https://doi.org/10.1136/bmjopen-2016-015447 PMID: 28615273

38. Zienkiewicz AK, Verschueren van Rees N, Homer M, Ong JJ, Christensen H, Hill D, et al. Agent-based modelling study of antimicrobial-resistant Neisseria gonorrhoeae transmission in men who have sex with men: towards individualised diagnosis and treatment. Sex Health. 2019;16(5):514-22. https://doi. org/10.1071/SH18235 PMID: 31476277

39. Hui BB, Ryder N, Su JY, Ward J, Chen MY, Donovan B, et al. Exploring the Benefits of Molecular Testing for Gonorrhoea Antibiotic Resistance Surveillance in Remote Settings. PLoS One. 2015;10(7):e0133202. https://doi.org/10.1371/journal. pone.0133202 PMID: 26181042

40. Tuite AR, Gift TL, Chesson HW, Hsu K, Salomon JA, Grad YH. Impact of Rapid Susceptibility Testing and Antibiotic Selection Strategy on the Emergence and Spread of Antibiotic Resistance in Gonorrhea. J Infect Dis. 2017;216(9):1141-9. https://doi. org/10.1093/infdis/jix450 PMID: 28968710

41. Toskin I, Govender V, Blondeel K, Murtagh M, Unemo M, Zemouri $C$, et al. Call to action for health systems integration of point-of-care testing to mitigate the transmission and burden of sexually transmitted infections. Sex Transm Infect. 2020;96(5):342-7. https://doi.org/10.1136/ sextrans-2019-054358 PMID: 32241905

42. Davies B, Turner KME, Frølund M, Ward H, May MT, Rasmussen $S$, et al. , Danish Chlamydia Study Group. Risk of reproductive complications following chlamydia testing: a populationbased retrospective cohort study in Denmark. Lancet Infect Dis. 2016;16(9):1057-64. https://doi.org/10.1016/S14733099(16)30092-5 PMID: 27289389

43. Golparian D, Rose L, Lynam A, Mohamed A, Bercot B, Ohnishi M, et al. Multidrug-resistant Neisseria gonorrhoeae isolate, belonging to the internationally spreading Japanese FC 428 clone, with ceftriaxone resistance and intermediate resistance to azithromycin, Ireland, August 2018. Euro Surveill. 2018;23(47). https://doi.org/10.2807/1560-7917. ES.2018.23.47.1800617 PMID: 30482267

44. Eyre DW, Sanderson ND, Lord E, Regisford-Reimmer N, Chau $\mathrm{K}$, Barker L, et al. Gonorrhoea treatment failure caused by a Neisseria gonorrhoeae strain with combined ceftriaxone and high-level azithromycin resistance, England, February 2018. Euro Surveill. 2018;23(27). https://doi.org/10.2807/1560-7917. ES.2018.23.27.1800323 PMID: 29991383

45. European Centre for Disease Prevention and Control (ECDC). Extensively drug-resistant (XDR) Neisseria gonorrhoeae in the United Kingdom and Australia. Stockholm: ECDC. 7 May 2018. Available from: https://ecdc.europa.eu/sites/portal/ files/documents/RRA-Gonorrhoea $\% 2$ C \% 20Antimicrobial\%20 resistance-United $\% 20$ Kingdom $\% 2 \mathrm{C} \% 20$ Australia.pdf

46. Public Health England (PHE). Surveillance of antimicrobial resistance in Neisseria gonorrhoeae. Key findings from the "Gonococcal resistance to antimicrobials surveillance programme' (GRASP) and related surveillance data. London: PHE; 2015. Available from: https://www.gov.uk/government/ uploads/system/uploads/attachment_data/file/476582/ GRASP_2014_report_final_111115.pdf

47. Huntington SE, Burns RM, Harding-Esch E, Harvey MJ, HillTout R, Fuller SS, et al. Modelling-based evaluation of the costs, benefits and cost-effectiveness of multipathogen point-of-care tests for sexually transmitted infections in symptomatic genitourinary medicine clinic attendees. BMJ Open. 2018;8(9):e020394. https://doi.org/10.1136/ bmjopen-2017-020394 PMID: 30201794

48. Monthly Average Rates London OFX. UKForex Limited. [Accessed: 9 Mar 2020]. Available from: https://www. ofx.com/en-gb/forex-news/historical-exchange-rates/ monthly-average-rates/

49. Office for National Statistics (ONS). Consumer price inflation time series. London: ONS. [Accessed: 9 Mar 2020]. Available from: https://www.ons.gov.uk/economy/ inflationandpriceindices/datasets/consumerpriceindices
License, supplementary material and copyright

This is an open-access article distributed under the terms of the Creative Commons Attribution (CC BY 4.0) Licence. You may share and adapt the material, but must give appropriate credit to the source, provide a link to the licence and indicate if changes were made.

Any supplementary material referenced in the article can be found in the online version.

This article is copyright of the authors or their affiliated institutions, 2020. 